\title{
UNA SELECCIÓN BIBLIOGRÁFICA SOBRE EL MÉTODO AICLE (APRENDIZAJE INTEGRADO DE CONOCIMIENTOS CURRICULARES Y LENGUA EXTRANJERA)
}

\begin{abstract}
Almudena Fernández Fontecha
RESUMEN. Este trabajo consiste en una bibliografía seleccionada sobre el método de enseñanza de segunda lenguas o lengua extranjeras a través de contenidos extralingüísticos conocido como Aprendizaje Integrado de Conocimientos Curriculares y Lengua Extranjera (AICLE). Su elaboración tiene como objetivo facilitar el trabajo de todos aqueIlos interesados en el método tanto desde un punto de vista teórico como desde un punto de vista práctico. El corpus básico de esta bibliografía consiste en una selección de artículos, capítulos de libros, libros, extractos de conferencias o simposios, videos y un listado de páginas web dedicadas al desarrollo de este método.

ABSTRACT. This work is a selected bibliography on the method of second/foreign language learning called Content and Language Integrated Learning (CLIL). Its main objective is to aid those who are interested in the theoretical part of the method as well as those interested in its implementation. The main corpus of this bibliography consists of a selection of articles, book chapters, books, reports from conferences and symposia, videos, and a list of websites dealing with this method.
\end{abstract}

\section{Introducción}

A la hora de comenzar cualquier tipo de investigación, sea cual sea el campo de estudio y el nivel académico, es de gran utilidad disponer de un listado de las referencias imprescindibles sobre el tema a investigar. Desafortunadamente no siempre se tiene la suerte de contar con tal listado. Así, en la mayoría de las ocasiones, la búsqueda bibliográfica se convierte en una verdadera odisea que tiene como protagonista al propio investigador/a.

Se hace, por lo tanto, necesario llevar a cabo la tarea de compilación de materiales (libros, artículos, tesis, monografías, páginas web, materiales audiovisuales, etc.) como elementos indispensables al menos en los estadios iniciales de una investigación. El investigador/a agradecerá enormemente poder hacer uso de listados que, aunque configurados por otros, representan la base sobre la que elegir, desdeñar o añadir otras referencias que servirán para personalizar y dar forma a la propia investigación.

Dentro del campo de Aprendizaje y Enseñanza de Segundas Lenguas, que es el que aquí nos concierne, podemos encontrar, entre otras, bibliografías generales (González y Asensio, 1996; Rodríguez, 1994), sobre los componentes del lenguaje (Tannacito, 1995), sobre materiales didácticos (Aronis, 1983), u organizadas en torno 
a un concepto determinado como, por ejemplo, la comunicación en segundas lenguas (Jiménez Catalán, 1999).

La que aquí ofrecemos es una lista de fuentes seleccionada que versa sobre un método concreto de enseñanza de segundas lenguas/lenguas extranjeras que, aunque presenta una gran diversidad terminológica (Content-based Language Teaching, Bilingual Education, Content-based Instruction, o Content and Language Integrated Learning -CLIL-, entre otros) ${ }^{1}$ consiste básicamente en la enseñanza de una segunda lengua o lengua extranjera a través de contenidos extralingüísticos.

\section{El método: Aprendizaje Integrado de Conocimientos curriculares y Lengua Extranjera (AICLE)}

En la actualidad, la Comisión Europea tiene entre sus objetivos principales el desarrollo de una política a favor del bilingüismo o multilingüismo en los países europeos. Para conseguir tal objetivo dicho organismo, en su Libro Blanco de Educación (1995), recomienda urgentemente la puesta en marcha en los países comunitarios de los métodos de enseñanza de lenguas que integran lengua y contenidos extralingüísticos, buscando fomentar aspectos de crucial importancia en el proceso enseñanza/aprendizaje como el aprendizaje significativo, la autonomía, la motivación o la atención del alumnado, entre otros. Se hace así eco de resultados satisfactorios obtenidos por estos métodos en diferentes contextos, principalmente bilingües (Canadá, EEUU, Gales, Finlandia, etc.). Autores como Mohan (1986), quien es considerado el padre del método, Brinton et al. (1989) o más recientemente Frufauf, Coyle, y Christ (1996), o Marsh y Langé (1999) apoyan su puesta en marcha y destacan su flexibilidad para introducirlo en contextos no bilingües.

A pesar de su vigente actualidad no se trata de un método nuevo. Sus orígenes se encuentran en el modelo de Inmersión, puesto en marcha a mediados de la década de los 60 en Quebec (Canadá), a la vez que guarda una estrecha relación con otros como, por ejemplo, Lengua a través del curriculum (Language Across the Curriculum), Lenguaje para propósitos especiales o para propósitos académicos (Language for Specific Purposes, Language for Academic Purposes). En todos ellos la forma lingüística deja de ser un fin en sí misma para convertirse en un mero medio de transmisión del significado, como sucede en situaciones de la vida real. Por lo tanto, en este método el aprendizaje de una lengua extranjera/segunda lengua es en gran medida un problema de proveer al alumnado de contenidos auténticos y motivadores.

\subsection{Tipos}

Existen algunas variaciones sobre la idea de enseñar una lengua a través de contenidos extralingüísticos. Esta variabilidad nos obliga a hacer alusión a diferentes métodos englobados bajo el término Aprendizaje Integrado de Conocimientos curriculares y Lengua Extranjera. Tal pluralidad Ileva implícita cierta flexibilidad a la hora

1. En nuestra exposición utilizaremos el término AICLE (Aprendizaje Integrado de Conocimientos curriculares y Lengua Extranjera), utilizado como equivalente del término en inglés CLIL (Content and Language Integrated Learning), uno de los términos que mejor definen la diversidad dentro del método aquí tratado. 
de adaptar el método a las circunstancias concretas de cada lugar, de tal manera que

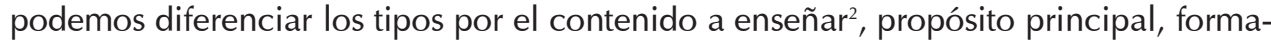
to, responsabilidades del profesorado, nivel de los estudiantes y tipo de evaluación, entre otros factores. Según estas diferencias Brinton et al. (1989) hacen alusión a tres tipos de modelos: Sheltered Subject Matter Teaching, Adjunct Model, y Theme-based Language Instruction. Así, como Met (1998) observa, se puede hablar de un continuum de tipos que engloba desde variantes más orientadas a la enseñanza del contenido extralingüístico hasta aquellas diseñadas con el fin claro de enseñar una segunda lengua o lengua extranjera.

\subsection{Postulados}

Los postulados de AICLE están en consonancia con estudios de psicología cognitiva y educativa, estudios de investigación en adquisición de segundas lenguas como los llevados acabo por Krashen (1985) o Cummins (1984), y conceptos como competencia comunicativa (Hymes, 1972), recogida actualmente en el Diseño Curricular Base.

Completamente integrados en la filosofía del Ilamado enfoque comunicativo el método AICLE presentan unos rasgos que lo definen, diferencian y a la vez lo unen a otros métodos englobados dentro de este enfoque. A continuación expondremos brevemente algunos de los principales postulados del método de Aprendizaje Integrado de Conocimientos curriculares y Lengua Extranjera:

- Naturalidad: Con este tipo de métodos se intenta crear unas condiciones de aprendizaje parecidas a las de la adquisición, como proceso inconsciente frente al aprendizaje, de la lengua materna en las que el sujeto se siente motivado por contenidos de tipo extralingüístico. Marsh (2000).

Esta forma indirecta de aprehender la lengua guarda una estrecha relación con otro método de creciente importancia en la actualidad, Aprendizaje por tareas (Taskbased Approach), mediante el cual el sujeto a través de la realización de tareas que poco o nada tienen que ver con el aspecto lingüístico aprende la segunda lengua o lengua extranjera.

- Incremento de la motivación: A la vez que el alumnado aprende en un entorno que, aunque formal, es adaptado para conseguir unas condiciones más naturales de aprendizaje, se fomentan factores con un importante peso dentro del aprendizaje en general como son la motivación o la atención.

- Uso de materiales auténticos: La autenticidad de los materiales es uno de los aspectos más controvertidos dentro del campo de Aprendizaje y Enseñanza de Segundas Lenguas. Lo ideal es asegurar el aprendizaje de la lengua manteniendo la autenticidad de los materiales seleccionados. Sin embargo, dicha autenticidad está reñida, en muchos casos, con la accesibilidad y comprensión de tales materiales por parte del sujeto. En su afán por impregnar la clase como contexto formal de aprendizaje de lo que acontece en el mundo exterior el método AICLE consi-

2. La diversidad de métodos de este tipo permite la enseñanza de la lengua a través de cualquier tema ya sea toda una asignatura curricular, parte de una asignatura curricular o un tema libre, entre otros. Esto permite resolver uno de los dilemas más acuciantes en el Sistema Educativo español como la enseñanza de los Temas Transversales, los cuales pueden ser considerados el input extralingüístico de la clase de Lengua Extranjera. 
dera la autenticidad de los materiales como uno de los factores más importantes dentro del proceso enseñanza/aprendizaje.

Sin embargo, como Van Lier (1996:144-145) observa, es posible la integración de materiales auténticos en clase si se hace uso de diferentes tipos de recursos para garantizar la accesibilidad de los mismos.

Algunos de ellos son ampliamente usados en los contextos AICLE. Entre otros podemos destacar el uso de materiales con información visual o el uso de diferentes tipos de gráficos u organizadores de la información (tablas, ciclos, redes, cuadros sinópticos, líneas del tiempo, etc.) Por medio de este tipo de recursos es posible el uso de materiales de diferente índole: periódicos, revistas, folletos, canciones, videos musicales, páginas web, películas, programas de televisión, extractos de novelas, cómics, poemas, o cualquier tipo de objetos de la vida cotidiana con algún tipo de contenido lingüístico como envases de productos alimenticios, folletos de instrucciones de aparatos eléctricos, etc.

Otros recursos contemplados por el método AICLE son el trabajo en grupo y la integración de las estrategias de aprendizaje. Con respecto al primero, este tipo de métodos fomenta la colaboración de los alumnos para resolver las actividades propuestas. Estas técnicas ayudan a disminuir la tensión que pueda existir en un entorno de aprendizaje formal a la vez que refuerza el desarrollo personal de los alumnos y alumnas, entre otros factores. (Kagan, 1987). Por otro lado, como algunas versiones del método son usadas para enseñar la lengua a través de otras asignaturas, sus defensores recomiendan la introducción de estrategias de aprendizaje que asistan al alumnado en el proceso de enseñanza/aprendizaje de la nueva lengua. La integración de estas estrategias es también recomendada en otras versiones que no exigen la enseñanza de asignaturas a través de la lengua extranjera o segunda lengua. Oxford (1990), Chamot y O’Malley (1994).

\section{Organización de la bibliografía}

A continuación describimos diferentes aspectos de tipo metodológico sobre la realización de la bibliografía que presentamos aquí.

\subsection{Propósito}

La idea de llevar a cabo esta recopilación de referencias sobre el método Aprendizaje Integrado de Conocimientos curriculares y Lengua Extranjera surge de la intención personal de promover dicho método facilitando el trabajo a cuantos estén interesados en el tema por cualquier motivo y a cualquier nivel.

Debe quedar claro que la aquí presentada es una bibliografía seleccionada, no se trata de una recopilación completa de fuentes sobre el método AICLE, debido a que es tremendamente ecléctico y hubiera sido una tarea casi imposible recoger cada referencia relacionada directa o indirectamente con el mismo. Sin embargo, a nuestro modo de ver, esta recopilación de fuentes responde ampliamente a las necesidades de un grupo amplio y heterogéneo de investigadores/as incluyendo al profesorado de idiomas de primaria, secundaria, bachillerato, universidad, alumnado de Filología Inglesa, de Magisterio de Lengua Extranjera, alumnado de Doctorado, y cualquier otra persona con cierto interés, teórico o práctico, en el método AICLE. 


\subsection{Corpus y criterios de selección}

Nuestra selección recoge en su mayoría artículos de revistas especializadas en Lingüística Aplicada y sobre todo en bilingüismo y multilingüismo. También incluye libros y capítulos de libros relacionados con diversos aspectos del método AICLE, asimismo hemos introducido extractos de conferencias o seminarios y una pequeña lista de páginas web relacionadas con el tema tratado.

\section{- Artículos}

Los artículos aquí adjuntados han sido extraídos de la siguiente selección de revistas:

- Annual Review of Applied Linguistics (vol. 1 -1980 / vol. 20 -1999)

- Bulletin suisse de linguistique appliquée (no 59 -1994 / nº 68 -1998)

- Cross-currents (vol. 4 -1977 / vol. 19 -1992)

- ELT Journal (vol. 36 -1981 / vol. 52 -1999)

- English for Specific Purposes (vol. 5 -1986 / vol.19 -2000)

- Foreign Language Annals (vol. 1 -1967 / vol. 32 -1999)

- Guidelines. A Periodical for Classroom (vol. 3 -1980 / vol. 20 - 1998)

- Journal of Multilingual and Multicultural Development (vol. 1 -1980 / vol. 18 -1997)

- TESL Canada Journal (vol. 1 -1984 / vol. 17 -1999)

- TESOL Journal (vol. 1 - 1992 / vol. 7 -1998)

- TESOL Quarterly (vol. 2 - 1968 / vol. 32 -1998)

- The Canadian Modern Language Review (vol. 31 -1974 / vol. 56 -2000)

- The CATESOL Journal (vol. 1, no 1 -1989 / vol 11, no 1 -1999)

Esta selección responde a los siguientes criterios: Por una parte todas las revistas coinciden en la temática, i.e. Aprendizaje y Enseñanza de Segundas Lenguas; por otra parte la mayoría son revistas en las que los artículos sobre bilingüismo y multilingüismo tienen especial cabida, lo cual es importante si se tienen en cuenta que el método AICLE surge y se desarrolla principalmente en contextos multilingües.

Con respecto al período temporal elegido, hemos querido reflejar la evolución de los métodos AICLE desde sus inicios hasta la actualidad.

\section{- Libros/capítulos}

Adjuntamos también libros que representan el trabajo de diferentes autores sobre el método aquí tratado así como capítulos específicos relacionados con el tema. Para su selección nos hemos basado en su aparición en los índices de otros libros o artículos que tratan este tema. Dentro de los libros seleccionados deben recibir una mención especial aquellos publicados recientemente por la Universidad de Jyväskylä (Finlandia), uno de los centros más prolíficos en el tratamiento del método AICLE. Se trata básicamente de libros teóricos sobre AICLE, programas de desarrollo del método o relatos de experiencias sobre su aplicación en el contexto europeo. 


\section{- Páginas web}

Hemos incluido también un pequeño apartado dedicado a páginas web esenciales en la búsqueda de información acerca del método AICLE. La selección está formada por páginas sobre proyectos específicos de AICLE, organismos europeos e instituciones con especial relevancia en el tema, etc. Aparte de este tipo de páginas incluimos la dirección de la base de datos ERIC, una de las más importantes en la búsqueda de referencias sobre cualquier tema e indicamos la forma de encontrar más información sobre el método al que nos referimos.

\section{- Otros}

Finalmente la bibliografía incluye algún extracto de conferencias o seminarios publicados, así como un par de videos llevados a cabo por profesionales del método en la Universidad de Jyväskylä (Finlandia).

La mayoría de estas fuentes se refiere a la lengua inglesa como segunda lengua o lengua extranjera, sin embargo las ideas teóricas o prácticas sobre el método son extrapolables a otras lenguas.

\subsection{Estructuración de las fuentes}

Con el ánimo de facilitar el trabajo a todas aquellas personas que hagan uso de esta bibliografía hemos procedido a organizarla en diferentes apartados, a su vez subdivididos en otros:

1. Información general o introductoria

2. Variaciones sobre el método

3. Formación del profesorado

- en general

- según niveles

- en la colaboración interdisciplinar

- en la evaluación

4. Destrezas lingüísticas

5. Recomendaciones y ejemplos para la aplicación del método

6. Materiales y recursos didácticos

- Autenticidad

- Música

- Literatura

- Periódicos

- Información audiovisual

7. La experiencia AICLE en Europa

8. Miscelánea

9. AICLE en la red

Todas las referencias incluidas se presentan en cada apartado en orden alfabético. Excepto en el caso de las páginas web, que se presentan en un apartado independiente, el resto de fuentes no se clasifican por tipos. 


\section{Presentación de la bibliografía}

\subsection{Información general o introductoria}

En este apartado se incluyen, por un lado, trabajos sobre investigaciones en Enseñanza y Aprendizaje de Segundas Lenguas que fundamentan la utilización del método como Krashen $(1982,1985)$ o Cummins $(1984)$; y por otro lado, aparecen trabajos pioneros y clave para el posterior desarrollo de las investigaciones sobre AICLE como los de Mohan (1979, 1986), Cantoni-Harvey (1987), Crandall (1987), Enright y McCloskey (1988) o Brinton, Snow y Wesche (1989), y otras referencias que recogen información general y básica sobre el método acerca de sus fundamentos teóricos, orígenes, evolución, postulados, tipos, distinciones terminológicas, relación con otros métodos, etc.

BAETENS-BEARDSMORE, H. (1996). Reconciling Content Acquisition and Language Acquisition in Bilingual Classrooms. Journal of Multilingual and Multicultural Development. 17:2-4, pp. 114-122.

BRAGGER, J. D., \& D. RICE. (1999). The Message Is the Medium: A New Paradigm for Content-Oriented Instruction. Foreign Language Annals, 32:3, p. 373.

BRENNAN, M. \& M. VAN NAERSSEN. (1988). Language and Content in ESP. ELT Journal, 43:3, pp. 196-205.

BRINTON, D. M., SNOW, M.A., \& M.B. WESCHE. (1989). Content-based second language instruction. Boston: Heinle and Heinle.

BYRAM, M. \& C. MORGAN. (1994). Teaching and learning language and culture. Clevedon: Multicultural Matters.

CANTONI-HARVEY, G. (1987). Content-Area Language Instruction: Approaches and strategies. Reading, MA: Addison-Wesley.

CHAMOT, A. U., \& J. M. O'MALLEY. (1987). The Cognitive Academic Language Learning Approach: A Bridge to the Mainstream. TESOL Quarterly, 21:2, p. 227.

CHAMOT, A., \& O'MALLEY, M. (1994). The CALLA Handbook: Implementing the Cognitive Academic Language Learning Approach. Reading, MA: Addison Wesley.

CHRISTIAN, D. et al. (1990). Combining Language and Content for SecondLanguage Students. En A. M. Padilla et al. Bilingual education. (p. 141). Newbury Park, CA: Sage.

CRANDALL, J., \& G. R. TUCKER. (1990). Content-based Language Instruction in Second and Foreign Languages. En S. Anivan (Ed.) Language Teaching Methodology for the Nineties. Singapore: RELC.

CRANDALL, J. A. (Ed.) (1987). ESL Through Content-Area Instruction. Englewood Cliffs, NJ: Prentice Hall Regents.

CUMMINS, J. (1984). Bilingualism and special education: Issues in the assessment and pedagogy. Clevedon, England: Multilingual Matters.

DE VALDÉS, M. E. (1977). Thematic Organization of Cultural Content in SecondLanguage Teaching. The Canadian Modern Language Review, 33:3, p. 354. 
DUNBAR, S. (1992). Integrating Language and Content: A Case Study. TESL Canada Journal, 10:1, pp. 62-70.

EARLY, M. \& G. M. TANG. (1991). Helping ESL Students Cope with Content-Based Texts. TESL Canada Journal, 8:2, pp. 34-44.

ENRIGHT D. S. \& M. MCCLOSKEY. (1988). Integrating English. Reading, Ma: Addison-Wesley.

JOHNS, A. M. (1992). What Is the Relationship Between Content-Based Instruction and English for Specific Purposes. The CATESOL Journal, 5:1, pp. 71-76.

KASPER, L.F. (1997). The Impact of Content-Based Instructional Programs on the Academic Progress of ESL Students. English for Specific Purposes, 16:4, pp. 309320.

KRASHEN, S. D. (1985) 1989. The Input Hypothesis: Issues and Implications. New York: Longman.

KRASHEN, S. D. (1982). Principles and Practice in Second Language Acquisition. Oxford: Pergamon Press.

KRUEGER, M., \& F. RYAN. (Eds.) (1993). Language and Content: Discipline- and Content-based Approaches to Language Study. Lexington, MA: D.C.Heath.

LEAVER, B. L. \& S. B. STRYKER. (1989). Content-Based Instruction for ForeignLanguage Classrooms. Foreign Language Annals, 22:3, pp. 269-275.

MARSH, D. (2000). Using Languages to Learn and Learning to Use Languages. An introduction to CLIL for parents and young people. TIE-CLIL. Disponible en la siguiente dirección: http://www.tieclil.org/HTML/products_E.html. (Febrero, 2001)

MARSH, D., C. ENNSER, \& D. SYGMUND. (1999). Pursuing Plurilingualism. Continuing Education Centre: University of Jyväskylä.

MCGROARTY, M. (1992). Cooperative Learning: The Benefits for Content-Area Teaching. En P. A. Richard-Amato and M. A. Snow. The multicultural classroom. Reading, MA: Addison-Wesley.

MET, M. (1991). Learning Language through Content: Learning Content through Language. Foreign Language Annals, 24:4, pp. 281-95.

MET, M. (1998). Curriculum Decision-making in Content-based Language Teaching. En J. Cenoz \& F. Genesee, (Eds.) Beyond Bilingualism: Multilingualism and Multilingual Education (pp. 35-63). Clevedon: Multilingual Matters.

MEYER, J. E. L. (1995). Role of Grammar Instruction in Thematic Content-Based EAP Courses. Guidelines. A Periodical for Classroom, 17:2, p. 6.

MOHAN B. A. (1990). Integration of Language and Content. En Proceedings of the First Research Symposium on Limited English Proficient Students' Issues (pp. 113-160). Washington, DC: U.S. Department of Education, Office of Bilingual Education and Minority Language Affairs (OBEMLA).

MOHAN, B. A. (1979). Relating Language Teaching and Content Teaching. TESOL Quarterly, 13:2, p. 171. 
MOHAN, B.A. (1986). Language and Content. Reading, MA: Addison-Wesley.

NIKULA, T. \& D. MARSH. (1998). Terminological Considerations Regarding Content and Language Integrated Learning. Bulletin suisse linguistique appliquée, 67, p. 13.

SNOW, M. A. (1991). Content-based Instruction: a Method with Many Faces. En J. Alatis (Ed.), Georgetown University Round Table in Language and Linguistics. Washington D. C.: Georgetown University Press.

SNOW, M. A. (1997). Trends and Issues in Content-Based Instruction. Annual Review of Applied Linguistics, 18, pp. 243-268.

SNOW, M. A., \& D. Brinton (Eds.), (1997). The Content-Based Classroom: Perspectives on Integrating Language and Content. White Plains, NY: Longman.

SPANOS, G. (1989). On the Integration of Language and Content Instruction. Annual Review of Applied Linguistics, 10, pp. 227-242.

SWAIN, M. (1988). Manipulating and Complementing Content Teaching to Maximize Second Language Learning. TESL Canada Journal, 6:1, pp. 68-83.

SWAIN, M. (1996). Integrating language and content in immersion classrooms: Research perspectives. The Canadian Modern Language Review, 52:4, p. 529.

TAYLOR, H. M. (1982). Teaching Content and Teaching English for Specific Purposes. Cross Currents, 9:2, p. 67.

WONG, K. (1992). What Do VESL and Content-Based Instruction Have in Common. The CATESOL Journal, 5:1, pp. 97-164.

\subsection{Variaciones sobre el método}

Esta sección comprende referencias que hace una alusión específica a las diferencias entre algunos de los tipos del método AICLE. Además de la selección que a continuación se expone se puede encontrar información general sobre estos tipos en referencias de la sección anterior como por ejemplo en Brinton, Snow y Wesche (1989) o Mohan (1986).

BURGER, S. \& J. DOHERTY. (1992). Sheltered Instructions for Young Adult Immigrants. En T. Irons \& S. Paribakht. Make changes, make a difference (p. 139) Welland: Soleil.

BURGER, S. (1989). Content-Based ESL in a Sheltered Psychology Course: Input, Output and Outcomes. TESL Canada Journal, 6:2, pp. 45-59.

CLÉMENT, R. et al. (1984). Second-Language Acquisition Through Subject-Matter Learning: A Study of Sheltered Psychology Classes at the University of Ottawa. The Canadian Modern Language Review, 41:2, pp. 268-282.

FREEMAN, D. et al. (1987). Success for LEP Students: The Sunnyside Sheltered English Program. TESOL Quarterly, 21:2, p. 361.

GOLDSTEIN, L. et al. (1994). Smiling Through the Turbulence: The Flight Attendant Syndrome and Other Issues of Writing Instructor Status in the Adjunct Model. The CATESOL Journal, 7:1, pp.19-30. 
IANCU, M. (1993). Adapting the Adjunct Model: A Case Study. TESOL Journal, 2:4, pp. 20-24.

KRASHEN, S. (1991). Sheltered Subject Matter Teaching. Cross Currents, 18:2, p. 183.

READY, D. \& M. WESCHE. (1992). An Evaluation of the University of Otawa's Sheltered Program: Language Teaching Strategies that Work. En R. J. Courchême, et al. Comprehension-based second language teaching (p. 389) Ottawa: University of Ottawa Press.

MURRAY, G. L. (1999). Autonomy, Technology, and Language-Learning in a Sheltered ESL Immersion Program. TESL Canada Journal, 17:1, pp. 1-15.

SNOW, M. A., \& D. M. Brinton. (1998). Content-Based Language Instruction: Investigating the Effectiveness of the Adjunct Model. TESOL Quarterly, 22:4, p. 553.

\subsection{Formación del profesorado}

Las características de las diferentes variantes del método AICLE exigen una formación previa de todos los profesores involucrados en su aplicación, sean de lengua extranjera o del resto de áreas curriculares. Esto permitirá, en la mayoría de los casos, mejorar la colaboración entre profesores de diferentes áreas así como delimitar el trabajo a llevar a cabo. Así, incluimos aquí una selección de referencias agrupadas en otras secciones que pueden ser de gran utilidad a la persona que decida aplicar el método en su contexto educativo:

\section{- en general}

Son referencias que tratan el tema de la formación del profesorado de una forma general e introductoria. De gran utilidad para aquellos interesados en la aplicación del método que necesiten una formación previa.

LAFAYETTE, R. C. (1993). Subject Matter Content: What Every Foreign Language Teacher Needs to Know. En G. Guntermann, et al. Developing language teachers for a change (p. 124) Lincolnwood, IL: National Textbook.

MARSH, D., T. NIKULA \& S. TAKALA. (1996). Teacher Training in Bilingual Education: Finland. En Teacher Training in Bilingual Education, Language Teacher Training and Bilingual Education (p. 27-47) The European Language Council: Berlin.

MASTER, P. (1992). What Are Some Considerations for Teacher Training in ContentBased Instruction. The CATESOL Journal, 5:1, pp. 77- 84.

RICHARD-AMATO, P. A., \& M. A. SNOW. (1992). Strategies for Content-Area Teachers. En P. A. Richard-Amato and M. A. Snow. The multicultural classroom (p. 145) Reading, MA: Addison-Wesley.

SAGLIANO, M. et al. (1998). Professional Training to Develop Content-Based Instruction in Higher Education. TESL Canada Journal, 16:1, pp. 36-51.

SEARS, C. (1998). Second language students in mainstream classrooms. A handbook for teachers in international schools. Clevedon: Multicultural Matters. 
SHORT, D. (1991). Content-Based English Language Teaching: A Focus On Teacher Training. Cross Currents, 18:2, p. 167.

SHORT, D. (1991). How to Integrate Language and Content Instruction: A Training Manual. Washington, DC: Center for Applied Linguistics.

\section{- según niveles}

En este apartado incluimos diferentes indicaciones sobre cómo tratar el método en diferentes niveles (Educación Infantil, Primaria, Secundaria, Bachillerato, Universidad, u otros).

BALLMAN, T. L. (1997). Enhancing Beginning Language Courses Through ContentEnriched Instruction. Foreign Language Annals, 30:2, p. 173.

BLANTON, L. (1992). A Holistic Approach to College ESL: Integrating Language and Content. ELT Journal, 46:3, pp. 285-293.

EARLY, M. (1991). Language and Content Learning K through 12. Cross Currents, $18: 2$, p. 179 .

English in the Content Areas. In Help! They Don't Speak English Starter Kit for Primary Teachers. 1998. Pp. 59-79. Eastern Stream Center on Resources and Training (ESCORT), Region IV Comprehensive Center at AEL, Region XIV Comprehensive Center/Center for Applied Linguistics. Disponible en la página: http://www.ael.org/cac/helpkit.htm. (Febrero, 2001)

FLOWERDEW, J. (1992). Content-Based Language Instruction in a Tertiary Setting. English for Specific Purposes, 12:2, pp. 121-38.

GAFFIELD-VILE, N. (1996). Content-Based Second Language Instruction at the Tertiary Level. ELT Journal, 50:2, pp. 108-114.

JAMES F., JR. VALENTINE \& L. M. REPATH-MARTOS. (1992). How Relevant Is Relevance?: An Examination of Student Needs, Interests, and Motivation in the Content-Based University Classroom. The CATESOL Journal, 5:1, pp: 25-42.

MOELLER, A. J. (1994). Content-Based Foreign Language Instruction in the Middle School: An Experiential Learning Approach. Foreign Language Annals, 27:4, pp. 535-544.

OXFORD, R. L. (1993). Progress in Tertiary Content-Based ESL Instruction. TESL Canada Journal, 11:1, p. 75.

PALMER, B. W. (1993). Eastern Michigan University's Programs in Language and International Business: Disciplines with Content. En M. Krueger and F. Ryan (Eds.) Language and content: Discipline- and content-based approaches to language study (p. 138) Lexington, MA: D. C. Heath.

SCOTT-TENNENT, Ch. (1997). La adquisición de lenguas extranjeras mediante contenidos en la ESO. En M. Pujol, y F. Sierra. (Eds.) Las lenguas en la Europa Comunitaria II (pp. 261-271) Amsterdam: Rodopi.

SHORT, D. J. (1994). Expanding Middle School Horizons: Integrating Language, Culture, and Social Studies. Tesol Quarterly, 28:3, pp. 581-608. 
SNOW, M. A. (1994). Collaboration Across Disciplines in Postsecondary Education: Attitudinal Challenges. The CATESOL Journal, 7:1, pp. 59-64.

\section{- en la colaboración interdisciplinar:}

En este apartado se incluye una selección de referencias relacionadas con la colaboración entre profesores de diferentes áreas o interesados en la integración de otro tipo de conocimientos y la enseñanza de una segunda lengua o lengua extranjera.

ALVERMANN, D. (1987). Strategic Teaching in Social Studies. En B. F. Jones et al. (Eds.) Strategic Teaching and Learning: Cognitive Instruction in the Content Areas (pp. 92-110). Alexandria, VA: Association for Supervision and Curriculum Development.

CARRASQUILLO, A. L., \& VIVIAN RODRÍGUEZ. (1995). Language Minority Students in the Mainstream Classroom. Clevedon: Multilingual Matters.

CLAIR, N. (1995). Mainstream Classroom Teachers and ESL Students. TESOL Quarterly, 29:1, p. 189.

CLEGG, J. (1996). Mainstreaming ESL. Case studies in integrating ESL students into the mainstream curriculum. Clevedon: Multilingual Matters.

CRANDALL, J. A. (Ed.), (1987). ESL Through Content-Area Instruction: Mathematics, Science and Social Studies. Englewood Cliffs, NJ: Prentice Hall.

GEE, Y. (1992). How Can ESL and Content Teachers Work Effectively Together in Adjunct Courses. The CATESOL Journal, 5:1, pp. 85-92.

HUDSON, T. (1991). A Content Comprehension Approach to Reading English for Science and Technology. TESOL Quarterly, 25:1, p. 77.

ISSERLIS, J. (1991). Content Area ESL: Social Studies. TESOL Quarterly, 25:1, p. 167.

LAGUNOFF, R. (1993). Life Science: Content and Learning Strategies. TESOL Quarterly, 27:1, p. 126.

OSTLER, C. (1998). Successful Vocabulary Learning in English for Business and Technology and other Content-Based Courses. Guidelines. A Periodical for Classroom, 20:1, p. 97.

RODRÍGUEZ, R. (1983). Earth and Physical Science: Content and Learning Strategies. Mary AnnChristison and Sharron Bassano. TESOL Quarterly, 27:1, p. 128.

ROSENTHAL, J. W. (1995). Teaching Science to language minority students. Clevedon: Multilingual Matters.

SAGLIANO, M., \& K. Greenfield. (1998). A Collaborative Model of Content-Based EFL Instruction in the Liberal Arts. TESOL Journal, 7:3, pp. 23-28.

STOOPS, L. (1998). How Content Teachers Interact With English Language Learners. TESOL Journal, 7:5, p. 24.

TANG, G. M. (1994). Teacher Collaboration in Integrating Language and Content. TESL Canada Journal, 11:2, pp.100-116. 
TEEMANT, A., et al. (1996). Collaborating with Content-Area Teachers: What Need to Share. TESOL Journal, 5:4, pp.16-20.

TERDY, D. (1986). Content Area ESL: Social Studies. Palatine, IL: Linmore.

\section{- en la evaluación}

Las aquí incluidas representan una pequeña muestra de referencias que tratan el tema de la evaluación cuando se integra la enseñanza de un tipo de conocimiento extralingüístico y la segunda lengua o lengua extranjera. Otros trabajos sobre la evaluación pueden ser encontrados por ejemplo en Snow and Brinton (1997:187-213) cuya referencia completa está en la sección 4.1. Información general o introductoria de esta bibliografía.

BROWN, J. D. (1991). Content-Based Language Testing. Cross Currents, 18:2, p. 174.

SHORT, D. J. (1993). Assessing Integrated Language and Content Instruction. TESOL Quarterly, 27:4, p. 627.

TURNER, J. L. (1992). Creating Content-Based Language Tests: Guidelines for Teachers. The CATESOL Journal, 5:1, pp. 43-58.

\subsection{Destrezas lingüísticas}

En esta sección incluimos básicamente artículos referentes al desarrollo de algunas de las destrezas del lenguaje (habla, escritura, lectura, escucha) al ser aplicado alguna variedad del método AICLE.

ARMBRUSTER, B. B. et al. (1990). Improving Content Area Reading Using Instructional Graphics (Technical Report NO. 508). Champaign: University of Illinois, Center for the Study of Reading.

EARLY, M. (1990). ESL Beginning Literacy: A Content-Based Approach. TESL Canada Journal, 7:2, pp. 82-93.

HERBER, H. L. (1970). Teaching Reading on Content Areas. Englewood cliffs, NJ: Prentice Hall.

KASPER, L. F. (1995). Theory and Practice in Content-Based ESL Reading. English for Specific Purposes, 14:3, pp. 223-230.

MARTIN, I. (1990). Vocabulary Acquisition in a Content-based Approach. Guidelines. A Periodical for Classroom, 12:2, p.1.

SHIH, M. (1986). Content-Based Approaches to Teaching Academic Writing. TESOL Quarterly, 20:4, p. 617.

VACCA, R., \& J. Vacca. (1993). Content-Area Reading. New York: Harper Collins.

\subsection{Recomendaciones y ejemplos para la aplicación del método}

A continuación presentamos una selección de referencias sobre actividades, modelos y formas específicas de llevar a cabo la integración del modelo en diferentes contextos. 
CELCE-MURCIA, M. (1989). Models for Content Based Curricula for ESL. The CATESOL Journal, 2:1, pp. 5-16.

COELHO, E. (1992). Integrating Language and Content. En C. Kessler. Cooperative language learning (p. 129) Englewood Cliffs, NJ: Prentice Hall Regents.

COYLE, D. (1999). Looking Fowards: Moving on in CEILINK International Bilingual Think Tank Report, Strasbourg, October 1998. Jyväskylä: Council of Europe (conference report).

ESKEY, D. E. (1992). Syllabus Design in Content-Based Instruction. The CATESOL Journal, 5:1, pp.11-24.

IRUJO, S. (1990). How to Plan Content-Based Teaching Units for ESL. ERIC Report 320452. Washington: ERIC Document Reproduction Service.

KIDD, R. and B. MARQUARDSON. (1997). The Foresee Approach to Integrated ESL Instruction. TESL Canada Journal, 15:1, pp.1-21.

LEIBMAN, A. (1992). Activities for the Pre-University Content-Based Classroom. Cross Currents, 19:2, p.185.

MARSH, D. \& B. MARSLAND. (Eds.) (1999). Learning with Languages. Continuing Education Centre: University of Jyväskylä.

MARSH, D. \& B. MARSLAND. (Eds.) (1999). CLIL Initiatives for the Millennium: Report on the CEILINK Think Tank. Continuing Education Centre: University of Jyväskylä.

MARSH, D., B. MARSLAND and T. NIKULA. (Eds.), (1997). Aspects of Implementing Plurilingual Education. Continuing Education Centre: University of Jyväskylä.

QUINCANNON, J., T. SOCIAS \& T. NAVÉS. (1999). Clever Consumers Investigate Detergents. Servei d'Innovació pedagógica i Ordenació Curricular. Departament d’Enseyament Generalitat de Catalunya.

SNOW, M. A., M. MYRIAM \& F. GENESEE. (1989). A Conceptual Framework for the Integration of Language and Content in Second/Foreign Language Instruction. TESOL Quarterly, 23:2, p. 201.

TAYLOR, S. K. (1998). Beyond belief: Variance in models of content-based instruction and school success among minority language learners. Bulletin suisse linguistique appliquée, 67, p. 61.

\subsection{Materiales y recursos didácticos}

Por una parte, este apartado está dedicado a la autenticidad que los teóricos del método AICLE recomiendan de los materiales a utilizar para la enseñanza de la segunda lengua o lengua extranjera. Por otra parte, adjuntamos una muestra de artículos que, aunque no tratan explícitamente de la práctica del método AICLE, están directamente relacionados con él ya que sus autores se refieren al uso de diferentes tipos de materiales (canciones, poemas, novelas, películas, periódicos, etc.) y de recursos didácticos para la aplicación de este método (televisión, radio, video, retroproyector, gráficos, etc.). 


\section{- autenticidad}

ABRAHAM, R. G. (1981).Teaching the English Verb System with Authentic Discourse. Cross Currents, 8:2, p. 23.

DRABBLE. G. (1981). Using Authentic Materials for Teaching Writing Skills. Guidelines. A Periodical for Classroom, 6, p. 41.

DUNLOP, I. (1981). Reading and communication: using authentic materials. World Language English, 1:1, p. 50.

DUNNETT, S. et al. (1987). The Effect of Authentic Materials in Acquiring a Second Language. The Canadian Modern Language Review, 43:3, pp. 479-492.

FABMY, J. J. AND BILTON. (1989). Using Authentic Texts: A Study Skills Module. Guidelines. A Periodical for Classroom, 11:2, p. 74.

GILLETT, A. J. (1981). Reading and communication: Using authentic materials. World Language English, 1:3, p. 200.

HOYT, H. (1981). Using Authentic Discourse in Teaching the Conditional. Cross Currents, 8:1, p. 1.

KE, C. \& S. MCGINNIS. (1992). Using Authentic Cultural Materials to Teach Reading in Chinese. Foreign Language Annals, 25:3, pp. 233.

MAINGAY, S. M. (1980). Selection and Grading of Authentic Material for the Reading Class. English Language Teaching Journal, 34:3, pp. 217-21.

PEACOCK, M. (1997). The Effect of Authentic Materials on the Motivation of EFL Learners. ELT Journal, 51:2, pp. 144-156.

SCHMIDT-RINEHART B. C. (1997). Authentic Materials and Mexican Immersion: A Professional Development Program Combining Pedagogy, Language, and Culture. Foreign Language Annals, 30:2, p. 201.

WONG, V., et al. (1995). Use of Authentic Materials at Tertiary Level. ELT Journal, 49:4, pp. 318-322.

\section{- música}

DOBBS, J. P. B. (1992). Music as Multicultural Education. En P. A. Richard-Amato and M. A. Snow. The multicultural classroom (p. 364) Reading, MA: Addison-Wesley.

DOMONEY, L. and S. HARRIS. (1993). Justified and Ancient: Pop Music in EFL Classrooms. ELT Journal, 47:3, pp. 234-241.

GRIFEE, D. (1988). Song and Music Techniques in Foreign and Second Language Classrooms. Cross Currents, 15:1, p. 23.

LOWE, A. S. (1998). L'enseignement de la musique et de la langue seconde: pistes d'intégration et conséquences pour les apprentissages. The Canadian Modern Language Review, 54:2, p. 218.

MCBEATH, N. (1986). Using Rock and Popular Music in EFL. Cross Currents, 13:1, p. 43. 
RICHARDS, J. (1969). Songs in Language Learning. TESOL Quarterly, 3:2, p.161.

SUBRAMANIYAN, N. (1985). The Use of Pop Songs in Language Learning. Guidelines. A Periodical for Classroom, 7:1, p. 79.

URBANCIC, A. \& J. VIZMULLER. (1981). Using Popular Music in the Foreign Language Classroom. The Canadian Modern Language Review, 38:1, pp. 81-87.

\section{- literatura}

BORETZ, M. et al. (1992). Realbooks: Literature as Content in ESL Classrooms. The CATESOL Journal, 5:1, pp. 59-70.

BRICE, S. (1996). Literature and Language Teaching: Re-creating Literature in the ESL Classroom. TESOL Quarterly, 30:4, p.776.

BRIEN, T. (1987). Learning Language through Literature, Learning Literature through Language exploiting a poem with adult language learners. Guidelines. A Periodical for Classroom, 9:1, p. 30.

BRINTON, D. (1990). The Literature-Enriched Curriculum. TESOL Quarterly, 24:4, p. 729.

CARTER, R. \& M. N. LONG. (1990). Testing Literature in EFL Classes: Tradition and Innovation. ELT Journal, 44:3, pp. 215-221.

CHIU, W. (1980). Dragons in the Nursery - Teaching English Through Nursery Rhymes and Popular Folksongs. Guidelines. A Periodical for Classroom, 4, p. 105.

CHRISTENSEN, B. (1990). Teenage Novels of Adventure as a Source of Authentic Material. Foreign Language Annals, 23:6, pp. 531-537.

CUSTODIO, B. \& M. J. Sutton. (1998). Literature-Based ESL for Secondary School Students. TESOL Journal, 7:5, p. 19.

DUNN, M. (1990). At the Door: Selected Literature for ESL Students, Sandra McKay and Dorothy Petitt. TESOL Quarterly, 24:4, p. 736.

ELLIOT, R. (1990). Encouraging Reader-Response to Literature in ESL Situations. ELT Journal, 44:3, pp. 191-198.

GAJDUSEK, L. (1988). Toward Wider Use of Literature in ESL: Why and How. TESOL Quarterly, 22:2, p. 227.

GAREIS, E. et al. (1998). Beyond Entertainment: Novels and Film Adaptations in the ESL/EFL Classroom. TESL Canada Journal, 15:2, p. 50.

HOLTEN, C. (1990). Literacy Through Literature. TESOL Quarterly, 24:4, pp. 730.

LAZAR, G. (1990). Using Novels in the Language-Learning Classroom. ELT Journal, 44:3, pp. 204-214.

LAZAR, G. (1994). Using Literature at Lower Levels. ELT Journal, 48:2, pp. 115-124.

LAZAR, G. (1996). Literature and Language Teaching: Exploring Literary Texts With the Language Learner. TESOL Quarterly, 30:4, p. 773. 
LEKI, I. (1983). Review: Jean S. Mullen, Outsiders: American Short Stories for Students of ESL and John F. Povey, Literature for Discussion: A Reader for Advanced Students of English as a Second Language. TESOL Quarterly, 18, p.729.

MALCOLM, I. G. \& K. MALCOLM. (1989). Literature and Authenticity - Advanced EFL Composition. Guidelines. A Periodical for Classroom, 11:2, p. 25.

MARSHALL, M. (1979). Love and Death in Eden: Teaching English Literature to ESL Students. TESOL Quarterly, 13:3, p. 331.

MCKAY, S. (1982). Literature in the ESL Classroom. TESOL Quarterly, 16:4, p.529.

MIRSKI, K., \& C. OSTLER. (1996). Using Lectures to Teach English for the Social Sciences in Vietnam. Guidelines. A Periodical for Classroom, 18:2, p.62.

OSTER, J. (1989). Seeing With Different Eyes: Another View of Literature in the ESL Class. TESOL Quarterly, 23:1, p.85.

PRESTON, W. (1982). Poetry Ideas in Teaching Literature and Writing to Foreign Students. TESOL Quarterly, 16:4, p. 489.

RAMSARAN, S. (1983). Poetry in the Language Classroom. ELT Journal, 37:1, pp. 36-43.

RONNQVIST, L. \& R. D. Sell. (1994). Teenage Books for Teenagers: Reflections on Literature in Language Education. ELT Journal, 48:2, pp.125-132.

ROSS, N. J. (1991). Literature and Film. ELT Journal, 45:2, pp.147-155.

ROSSER, C. (1995). Anne Frank: A Content-Based Research Class. TESOL Journal, 4:4, pp. 4-6.

SEOK, C. (1987). Exploiting a poem with adult language learners Tony O'Brien Using Local Literature to Language Teaching. Guidelines. A Periodical for Classroom, 9:1, p. 40.

SPACK, R. (1985). Literature, Reading, Writing, and ESL: Bridging the Gaps. TESOL Quarterly, 19:4, p. 703.

SUBRAMANIYAN, N. (1990). The Short Story in the Language Classroom. Guidelines. A Periodical for Classroom, 12:2, p. 22.

WIDDOWSON, H. G. (1983). Talking Shop: On Literature and ELT. ELT Journal, 37:1, pp. 30-35.

WILLOQUET-MARICONDI, P. (1992). Integrating ESOL Skills through Literature. TESOL Journal, 1:2, pp. 11-14.

YANG, W. L. Y. (1976). Teaching Chinese Through Chinese Literature. The Modern Language Journal, 60:1-2, pp.31.

\section{- periódicos}

LATCHFORD, C. H. (1973). Newspapers: Vehicles for Teaching ESOL with a Cultural Focus. TESOL Quarterly, 7:2, p.145.

MAYER, M. (1988). Newspaper Summaries: An Integrated Skills Activity. Cross Currents, 14:2, p. 58. 
MCGHIE, C. (1990). Hof Off the Press: Using Today's Newspaper in the Classroom. Guidelines. A Periodical for Classroom, 12:1, p. 44.

NIELSON, D. (1983). Using Short Newspaper Articles with ESL Students. Cross Currents, 10:2, p. 92.

SMITH, M. F. (1984). Using English Language Newspapers in First-Year Foreign Language Classes. Foreign Language Annals, 17:1, pp. 43-46.

\section{- información audiovisual}

ANDERSON, E. A. (1984). Using Radio and Television to Learn English. Guidelines. A Periodical for Classroom, 6:1, p. 8.

AREY, M. J. (1993). French Films: Pre-Texts for Teaching Syntax. Foreign Language Annals, 26:2, pp. 252-264.

BURKE, E. V. (1980). Pictorial Aids. Guidelines. A Periodical for Classroom, 4, p. 29.

CHAMBERLAIN, A. (1980). The Impact of Audio-Visual Aids in the Language Classroom. Guidelines. A Periodical for Classroom, 4, p. 1.

CHEW, P. (1980). What's in a Painting? A Look at an Educational Television Lesson. Guidelines. A Periodical for Classroom, 4, p. 63.

COOK, J. (1996). The Art of Observation: Developing the Writing Process through Film. TESOL Journal, 5:2, pp. 6-8.

DAHMS, R. G. (1974). Films in the Foreign Language Program. The Canadian Modern Language Review, 31:1, p. 66.

DODDS, D. (1997). Using Film To Build Writing Proficiency in a Second-Year Language Class. Foreign Language Annals, 30:1, pp. 140-147.

EVANS, J. (1984). Video and Nonverbal Communication. Guidelines. A Periodical for Classroom, 6:1, p. 25.

GAREIS, E. (1997). Movies in the Language Classroom: Dealing with Problematic Content.. TESOL Journal, 6:4, pp. 20-23.

IRVING, J. S. (1976). Cassettes, Films, and the Partner System: Adding a New Dimension to the Conversation Class. Foreign Language Annals, 9:4, pp. 284-288.

KAUR, S. (1984). An Introduction to the Use of Video in Oral Language Teaching. Guidelines. A Periodical for Classroom, 6:1, p. 14.

KITAO, K. (1986). Using Authentic Video Materials in the Language Classroom. Cross Currents, 12:2, p.17.

KREIDLER, C. J. (1971). Effective Use of Visual Aids in the ESOL Classroom. TESOL Quarterly, 5:1, p. 19.

MOLLICA, A. (1976). Cartoons in the Language Classroom. The Canadian Modern Language Review, 32:4, p. 424.

MOLLICA, A. (1978). The Film Advertisement: A Source for Language Activities. The Canadian Modern Language Review, 34:2, p. 221. 
MOLLICA, A. (1979). A Tiger in Your Tank: Advertisements in the Language Classroom. The Canadian Modern Language Review, 35:4, p. 691.

NEWMAN, J. (1998). Film Is Content: A Study Guide for the Advanced ESL Classroom. Julia A. Williamson and Jill C. Vincent. TESOL Quarterly, 32:1, p. 165.

OMAGGIO, A. C. (1979). Pictures and Second Language Comprehension: Do They Help? Foreign Language Annals, 12:2, pp.107-116.

PLAISTER, T. (1980). Using the OHP to Teach from Crossword Puzzles and Clozed Passages. Guidelines. A Periodical for Classroom, 4, 1980, p. 54

PORTER, D. \& J. ROBERTS. (1981). Authentic Listening Activities. ELT Journal, 36:1, pp. 37-47.

SANDSBERRY, L. (1979). Magazine Ads and Logic in the ESL Classroom. TESOL Quarterly, 13:4, 1979, p. 501.

SARAWIT, M. (1984). The VTR - A Communicative Activity Aid. Guidelines. A Periodical for Classroom, 6:1, p. 21.

SMITHIES, M. (1982). From Graphics to Connected Prose. Guidelines. A Periodical for Classroom, 4:1, p. 42 .

SUBRAMANIYAN, N. (1987). The Radio News as a Resource for Language Learning. Guidelines. A Periodical for Classroom, 9:1, p. 45.

SWAN, J. (1980). Teaching Lecture Note-Taking with the Aid of the OHP. Guidelines. A Periodical for Classroom, 4, p. 47.

SWAN, J. (1982). Teaching Note-Making from Books with the Aid of Two Overhead Projectors. Guidelines. A Periodical for Classroom, 4:1, p. 29.

TANG, G. M. (1991). The Role and Value of Graphic Representation of Knowledge Structures in ESL Student Learning: An Ethnographic Study. TESL Canada Journal, 9:1, pp. 29-41.

TERRY, K. A. (1980). Audio-Visual Considerations. Guidelines. A Periodical for Classroom, 4, p. 23.

TREMBLAY, R. (1978). Using Magazine Pictures in the Second-Language Classroom. The Canadian Modern Language Review, 35:1, p. 82.

TURTON, N. D. (1980). Teaching Fluency Through Cartoons. Guidelines. A Periodical for Classroom, 4, p.101.

VOLLER, P., \& S. WIDDOWS. (1993). Feature Films as Text: A Framework for Classroom Use. ELT Journal, 47:4, 1993, pp. 342-353.

WANADILOK, K. (1980). Towards More Effective Language Teaching Through Educational Films. Guidelines. A Periodical for Classroom, 4, p. 59.

\section{7. La experiencia AICLE en Europa}

La siguiente selección es una muestra de experiencias sobre el método AICLE en diferentes países europeos. Incluimos además las referencias de dos vídeos sobre el 
método publicados por la Universidad de Jyväskylä (Finlandia) así como la referencia del Libro Blanco sobre Educación publicado por la Comunidad Europea en 1996 y que trata de la política que se está llevando a cabo para la introducción y aplicación del método en Europa.

BAETENS-BEARDSMORE, H. (Ed.), (1993). European Models of bilingual education. Clevedon: Multilingual Matters.

CENOZ, J. \& U. JESSNER. (2000). English in Europe. Clevedon, Philadelphia, Toronto, Sydney, Johannesburg: Multilingual Matters.

EUROPEAN COMMISSION. (1996). White Paper - Teaching and Learning: Towards the Learning Society. (based on COM (95) 590 final, 29 November 1995), Luxembourg: Office for Official Publications of the European Communities.

FRUFAUF, G., , D. COYLE. \& I. CHRIST. (Eds.), (1996). Teaching Content in a Foreign Language. Practice and Perspectives in European Bilingual Education. Alkmaar: European Platform for Dutch Education.

Intertalk: Plurilingual Education across Europe. (1997). Video, producido con el apoyo de la Comisión Europea. Continuing Education Centre: University of Jyväskylä.

MARSH, D. \& G. LANGÉ. (Eds.), (1999). Implementing Content and Language Integrated Learning. Continuing Education Centre: University of Jyväskylä.

MARSH, D., B. MARSLAND, \& A. MALJERS. (Eds.), (1998). Future Scenarios in Content and Language Integrated Learning. Continuing Education Centre: University of Jyväskylä.

Teaching with Foreign Languages. (1988). Video, emitido en la BBC en "The Learning Zone". Continuing Education Centre: University of Jyväskylä.

THÜRMANN, E., \& H. HELFRICH. (Eds.), (1993). Language Learning for European Citizenship. Report on Workshop 12 A. Bilingual Education in Secondary Schools: Learning and teaching non-language subjects through a foreign language. Strasbourg: Council of Europe.

\subsection{Miscelánea}

A continuación incluimos otras referencias sobre el método AICLE que por su temática no pueden incluirse en ninguno de los grupos anteriores. Se trata de un grupo heterogéneo de referencias que tratan de aspectos tan diversos como la aplicación del método AICLE en la enseñanza de adultos, experiencias puntuales de aplicación del método o bilingüismo, entre otros.

ADAMSON, H. D. (1993). Academic Competence: Theory and Classroom Practice Preparing ESL Students for Content Courses. New York: Longman.

CRANDALL, J. (1992). Content-Centered Learning in the U.S. Annual Review of Applied Linguistics, 13, pp.111-126.

HARKLAU, L. (1994). ESL Versus Mainstream Classes: Contrasting L2 Learning Environments. TESOL Quarterly, 28:2, p. 241. 
MAYER, M., \& L. MAYER. (1991). 4 Years Later-Are We Content With Content? Cross Currents, 18:2, p. 206.

MILK, R. (1990). Integrating Language and Content: Implications for Language Distribution in Bilingual Classrooms. En R. Jacobson \& C. Faltis (Eds.), Language Distribution Issues in Bilingual Schooling (pp. 32-44) Clevedon: Multilingual Matters.

OVERFIELD, D. M. (1997). From the Margins to the Mainstream: Foreign Language Education and Community-Based Learning. Foreign Language Annals, 30:4, pp. 485-491.

SHAW, S. L. (1992). The English Connection: A Content-Based Grammar and Discussion Text. Gail Fingado, Leslie J. Freeman, Mary Reinbold Jerome, and Catherine Vaden Summers. TESOL Quarterly, 26:4, p. 755.

STERNFELD, S. (1991). Exploring Community in a Content-Based Foreign Language Classroom. Cross Currents, 18:2, p.143.

SWAIN, M. \& L. MICCOLI. (1994). Learning in a Content-Based, Collaboratively Structured Course: The Experience of an Adult ESL Learner. TESL Canada Journal, 12:1, pp. 15-28.

\subsection{AICLE en la red}

Hemos querido incluir esta sección como parte importante de la bibliografía con el fin de proponer una forma de actualizar la selección bibliográfica aquí incluida poniendo en contacto al interesado con los organismos, instituciones, redes, comités, proyectos, etc. relacionados con el método AICLE.

\section{http://www.euroclic.net}

Se trata de una de las páginas web más importantes sobre AICLE en Europa. Está coordinada por la Plataforma Europea para la Educación Holandesa con sede en La Haya (Holanda), por la Universidad de Jyväskylä (Finlandia), y por la Oficina Regional de Bilingüismo, con sede en Estrasburgo (Francia). Desde la página se pueden bajar diferentes artículos del boletín que sus responsables publican. Existe un foro donde se intercambia información sobre cursos, seminarios, materiales, etc. sobre AICLE. Además cuenta con un banco de materiales (libros, videos, bibliografías, etc.) que, sin embargo, al cierre de este artículo, se encuentra en construcción.

Esta página ofrece links a otras páginas interesantes como por ejemplo:

http://www.netwerktto.europeesplatform.nl/eng index.html: Página web de la Plataforma Europea para la Educación Holandesa.

http://www.jyu.fi/: Página de la Universidad de Jyväskylä (Finlandia)

http://olaf.hiof.no/ glenoh/CLIL-network.html: Página de AICLE en la Higher Education Research Network.

http://skol1.telia.se/saint/: Página de la Asociación Sueca de Profesores Bilingües y de Inmersión. 
http://www.geocities.com/bildnott/index.htm: Página web de BILD (Bilingual Integration of Languages and Disciplines) referente a una serie de projectos sobre la enseñanza de asignaturas a través de una lengua extranjera en la Universidad de Nottingham.

http://www.tieclil.org/: Página del proyecto Lingua A sobre la integración de contenido y lenguaje en Europa. (Translanguage in Europe -Content and Language Integrated Learning).

http://ericir.syr.edu/: Dirección de la página web de ASKERIC a través de la que se puede acceder a la base de datos de ERIC donde se pueden encontrar todo tipo de referencias sobre cualquier tema. Una de las mejores formas de conseguir información sobre AICLE es introduciendo content area teaching en la opción Identifiers. Se accederá a más de 600 entradas relacionadas de alguna forma con el método del que hemos tratado en este artículo.

\section{Referencias Bibliográficas}

ARONIS, C. (1983). Annotated Bibliography of ESL Materials. Teachers of English to Speakers of Other Languages. Washington DC: Teachers of English to Speakers of Other Languages.

BRINTON, D. M. ET AL. (1989). Content-based second language instruction. Boston: Heinle and Heinle.

CHAMOT, A., \& M. O'MALLEY. (1994). The CALLA Handbook: Implementing the Cognitive Academic Language Learning Approach. Reading, MA: Addison Wesley.

CUMMINS, J. (1984). Bilingualism and special education: Issues in the assessment and pedagogy. Clevedon, England: Multilingual Matters.

EUROPEAN COMMISSION. (1996). White Paper - Teaching and Learning: Towards the Learning Society. (based on COM (95) 590 final, 29 November 1995), Luxembourg: Office for Official Publications of the European Communities.

FRUFAUF, G., D. COYLE, \& I. CHRIST. (1996). Teaching Content in a Foreign Language. Practice and Perspectives in European Bilingual Education. Alkmaar: European Platform for Dutch Education.

GONZÁLEZ, J. L. y M. ASENSIO. (1996). Guía documental sobre aprendizaje en segundas lenguas y lenguas extranjeras. Infancia y aprendizaje, 75, pp. 75:83.

HYMES, D. (1972). "On Communicative Competence". En: PRIDE, J.B. and J. HOLMES. (Eds.) Sociolinguistics. Harmondsworth: Penguin.

JIMÉNEZ CATALÁN, R. M. (1999). Lingüística aplicada al aprendizaje y enseñanza del inglés. Perspectivas, fuentes y guía de prácticas. Logroño: Universidad de La Rioja. Servicio de Publicaciones.

KAGAN, S. (1987). Cooperative learning resources for teachers. Riverside, CA: University of California.

KRASHEN, S. (1985) 1989. The Input Hypothesis: Issues and Implications. New York: Longman. 
MARSH, D. (2000). Using Languages to Learn and Learning to Use Languages. An introduction to CLIL for parents and young people. TIE-CLIL. Disponible en la siguiente dirección: http://www.tieclil.org/HTML/products_E.html. (Febrero, 2001)

MARSH, D. \& G. LANGÉ. (1999). Implementing Content and Language Integrated Learning. Continuing Education Centre: University of Jyväskylä.

MET, M. (1998). Curriculum Decision-making in Content-based Language Teaching. En J. Cenoz and F. Genesee. (Eds.) Beyond Bilingualism: Multilingualism and Multilingual Education (pp. 35-63) Clevedon: Multilingual Matters..

MOHAN, B. A. (1979). Relating Language Teaching and Content Teaching. TESOL Quarterly, 13:2, p. 171.

MOHAN, B.A. (1986). Language and Content. Reading, MA: Addison-Wesley.

OXFORD, R.L. (1990). Language Learning Strategies. New York: Newbury House.

RODRÍGUEZ, T. (1994). Bibliografía sobre enseñanza/aprendizaje de inglés lengua extranjera. Aula Abierta, 64, pp.237-249.

TANNACITO, (1995). A Guide to Writing in English as a Second or Foreign Language: An Annotated Bibliography of Research and Pedagogy. Virginia, USA: Teachers of English to Speaker of Other Languages (TESOL), Inc.

VAN LIER, L. (1996). Interaction in the Language Curriculum: Awareness, Autonomy and Authenticity. London, New York: Longman. 